\title{
Conformal heating/cooling channels design in rapid heat cycle molding process
}

\author{
Fatma Kria a ${ }^{\text {a }}$ Moez Hammami And Mounir BacCar \\ National School of Engineers of Sfax (ENIS), Research Unit of Computational Fluid Dynamics and Transfer \\ Phenomena (CFDTP), B.P. 1173, Sfax, Tunisia
}

Received 14 July 2015, Accepted 13 may 2016

\begin{abstract}
A three-dimensional study of the thermal regulation system of Rapid Heat Cycle Molding process producing complex-shaped automotive part was undertaken. This numerical study aims at improving the design of thermal control system so as to improve the quality of the molded part and the process productivity. The thermal responses in mold and cavity are predicted by the commercial Finite Volume Analysis software Fluent 6.3.26 in cyclic transient regime. It was shown that a regular regime is established from the second molding cycle. Besides, the three dimensional thermal responses have shown the limitations of the classic conformal design in terms of temperature uniformity and cycle time for the complex shaped automotive part. Hence, and based on conventional manufacturing process, the efficiency of a new conformal design has been shown. In fact, simple drillings coupled with concentric tubes are able to produce complicated conformal heating/cooling channels, which are easier to fabricate, assemble and which have low manufacture cost compared with other methods. By this proposed new conformal design, we could greatly reduce the cycle time, temperature gap and energy consumption.
\end{abstract}

Key words: Rapid heat cycle molding / classic conformal design / new conformal design / thermal control / numerical simulation

\section{Introduction}

Since the injection molding technology is among the most important industrial processes in the production of thermoplastic parts, improving the quality of thermoplastic products and reducing the cycle injection time have become the primary objective of processors.

Regarding the conventional injection molding (CIM), several research works have been concerned with the design of conformal cooling channels. With these cooling systems, used especially for products with complicated geometry, channels design should fit the shape of the molded part. In fact, Hassan et al. [1] have studied the effect of the position and the form of cooling channels on the (CIM) efficiency. They have found that as the cooling channels follow the profile of the product, the cooling time was reduced and the thermal distributions throughout the polymer were improved. Moreover, Agazzi et al. [2] have proposed methodology with two steps for the design of the cooling system. Firstly, they have used an objective function based on the part quality and process productivity to find the optimal temperature distribution in the plastic part. Secondly, they have used the

\footnotetext{
${ }^{a}$ Corresponding author: fatmakria1984@yahoo.fr
}

optimal solution to determine the design of the cooling channels. Shayfull et al. [3] have proposed 3D conformal cooling system formed by milled grooves with square shape (MGSS) to product front panel housing. These authors [3] have compared their proposed cooling system with straight drilled (circular) cooling channels, and they have demonstrated that Milled Groove Square Shape (MGSS) method is more efficient in cooling phase. Furthermore, Wang et al. [4] have proposed cooling system, based on product contour, for CIM producing helmets and cell phones. They have shown that this strategy can reduce the cooling time, promote uniform temperature and reduce volumetric shrinkage of the finished products.

Conformal channels can be manufactured by U-shape milled groove [5] or 3D printing [4,6,7]. Nevertheless, the cost of $3 \mathrm{D}$ printing is still very expensive, especially for large injection mold, which limits the application of this manufacturing method.

However, despite the development of conventional injection molding (CIM), several problems remain still unavoidable specifically those related to the mold cost and products quality such as weld lines, flow marks, poor surface quality [8] and shrinkage $[9,10]$. To overcome these problems, many authors [11-14] have suggested the 


\section{Nomenclature}

\begin{tabular}{|ll|}
\hline$C_{\mathrm{p}}$ & Heat capacity $\left(\mathrm{J} \cdot \mathrm{kg}^{-1} \cdot{ }^{\circ} \mathrm{C}^{-1}\right)$ \\
$D$ & Channel diameter $(\mathrm{m})$ \\
$g$ & Gravity acceleration $\left(\mathrm{m} \cdot \mathrm{s}^{-2}\right)$ \\
$h_{\mathrm{h}}$ & Heat transfer coefficient for heating stage $\left(\mathrm{W} \cdot \mathrm{m}^{-2} \cdot{ }^{\circ} \mathrm{C}^{-1}\right)$ \\
$h_{\mathrm{c}}$ & Heat transfer coefficient for cooling stage $\left(\mathrm{W} \cdot \mathrm{m}^{-2} \cdot{ }^{\circ} \mathrm{C}^{-1}\right)$ \\
$H_{\text {liq }}$ & Liquefaction latent heat of water $\left(\mathrm{J} \cdot \mathrm{kg}^{-1}\right)$ \\
$L$ & Length of vertical heating channel $(\mathrm{m})$ \\
$n$ & Normal direction \\
$T$ & Temperature $\left({ }^{\circ} \mathrm{C}\right)$ \\
\hline \multicolumn{2}{c|}{ Greek symbols } \\
\hline$\lambda$ & Thermal conductivity $\left(\mathrm{W} \cdot \mathrm{m}^{-1} \cdot{ }^{\circ} \mathrm{C}{ }^{-1}\right)$ \\
$\mu$ & Dynamic viscosity $\left(\mathrm{kg} \cdot \mathrm{m}^{-1} \cdot \mathrm{s}^{-1}\right)$ \\
$\rho$ & Density $\left(\mathrm{kg} \cdot \mathrm{m}^{-3}\right)$ \\
\hline \multicolumn{2}{c}{ Dimensionless numbers } \\
\hline $\operatorname{Re}$ & Reynolds number \\
$\mathrm{Pr}$ & Prandtl number \\
\hline & \\
\hline $\mathrm{m}$ & Mold \\
$\mathrm{p}$ & Polymer \\
$\mathrm{s}$ & Steam \\
$\mathrm{w}$ & Water \\
\hline
\end{tabular}

preheating of the mold before each filling phase. The preheating of the cavity surface at an appropriate temperature limits the polymer thermal losses by contact with cavity during the filling phase and then permits to conserve low viscosity of the fluid. Therefore, the mold cyclic heating facilitates polymer flow to replicate surface more successfully. Thus, thermal cycling prevents appearance of flow marks, weld lines and poor surfaces quality. So, a new thermoplastic injection process called rapid heat cycle molding (RHCM), developed by Ono Sangyo Co. Ltd. Cooperating with Mitsu Chemical Co. Ltd [15], has been proposed. This method consists of a rapid heating of the mold until reaching the glass transition temperature of the polymer, before filling the mold. Then mold should be cooled to a temperature permitting the ejection of the finished product without any deformation. The two key technical indicators of the RHCM process are the heating/cooling rapidity of thermal control system ensuring high process productivity and temperature uniformity at the cavity surface providing good products quality.

Previous experimental works [11-14] have investigated the influence of temperature increase at the cavity surface on the appearance of the injected part. They have shown that an adequate cavity surface temperature eliminates the prematurely freezing of the polymer during the filling phase, and improves the replication, giving an excellent aesthetics and more gloss.

Therefore, the heating phase has a major importance in the RHCM process. That is why several heating techniques, namely electric heating [16, 17], infrared heating [18] and steam heating [19] have been studied.
The design of the thermal regulation system is of great importance to improve the RHCM process. Then, several works $[15,20,21]$ have optimized the layout of the heating/cooling channels to minimize the required heating time and the deviation of the cavity surface temperature. Li et al. [15] have studied in 2D RHCM mold for the production of LCD TV panel using genetic algorithm. In their works, the optimization of the distance between heating channels improves the temperature uniformity leading to a good surface state. Wang et al. [20] have used the particle swarm optimization (PSO) method to study the $2 \mathrm{D}$ layout of thermal regulation system for producing a rectangular part. To perform the heating efficiency and temperature distribution uniformity, authors [20] have optimized the geometric parameters including the diameter of heating/cooling channels, distances channels-cavity and path between adjacent channels. Wang et al. [21] have considered the optimization of the design of the heating/cooling channels of Water-assisted Rapid Heat Cycle Molding (WRHC) process for the production of air conditioning panel. They have fixed the number and the diameter of channels and have optimized the distance from the cavity surface to the center of the channels, and the distances between adjacent channels by using a two-stage multi-objective optimization approach.

In spite of the development of research interested in the optimization of the design of thermal control system, the majority of these works have investigated in $2 \mathrm{D}$, only the first molding cycle. However, Hammami et al. [22] have studied a 3D thermal regulation system of RHCM producing LCD panel and they have found a large difference between the first cycle and the steady cycle. They have shown that the necessary time and the consumed energy for a heating phase can be reduced by about $50 \%$ after the first cycle molding, which can be explained by the thermal inertia of the massive central part of the mold.

Others 3D works, are interested in the study of simple shape product (specimens [23] or cover plate [24]). For part with complex geometry, Wang et al. [25] have investigated two different designs of heating channels for RHCM application. The first system includes straight parallel channels. The second design has been developed using baffles. By comparing the heating systems, they [25] have demonstrated an improvement of $27.3 \%$ in the heating efficiency.

For the aforementioned reasons and based on conventional machining processes, we will prove that accordingly to the morphology of the part, it is possible to generate conformal heating/cooling channels, particularly for complex shaped automotive parts. The method consists of designing a heating/cooling channels system, by keeping a nearly uniform distance between the center of the heating/cooling channels and the mold cavity, offering a better temperature homogenization on the surface enveloping the plastic part and reducing the cycle time.

The purpose of this numerical study is to improve the thermal control system so that the quality of the part and the productivity of the RHCM process are perfected. To achieve this objective, several arrangements of 

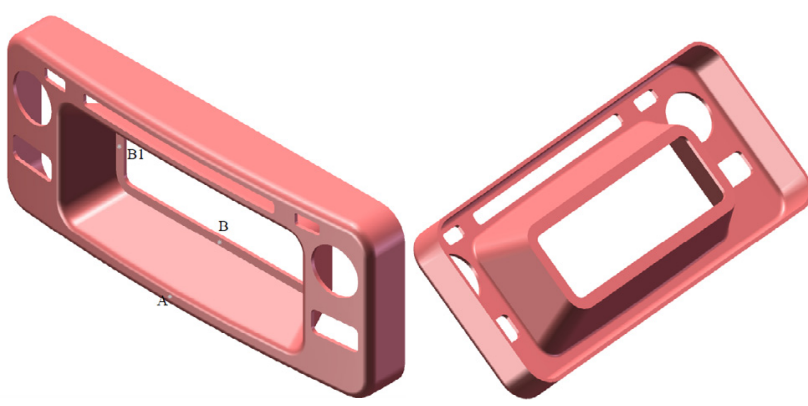

(a)

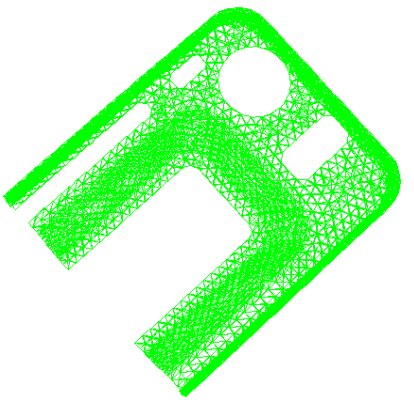

(b)

Fig. 1. Automotive part. (a) 3D model, (b) computational domain.

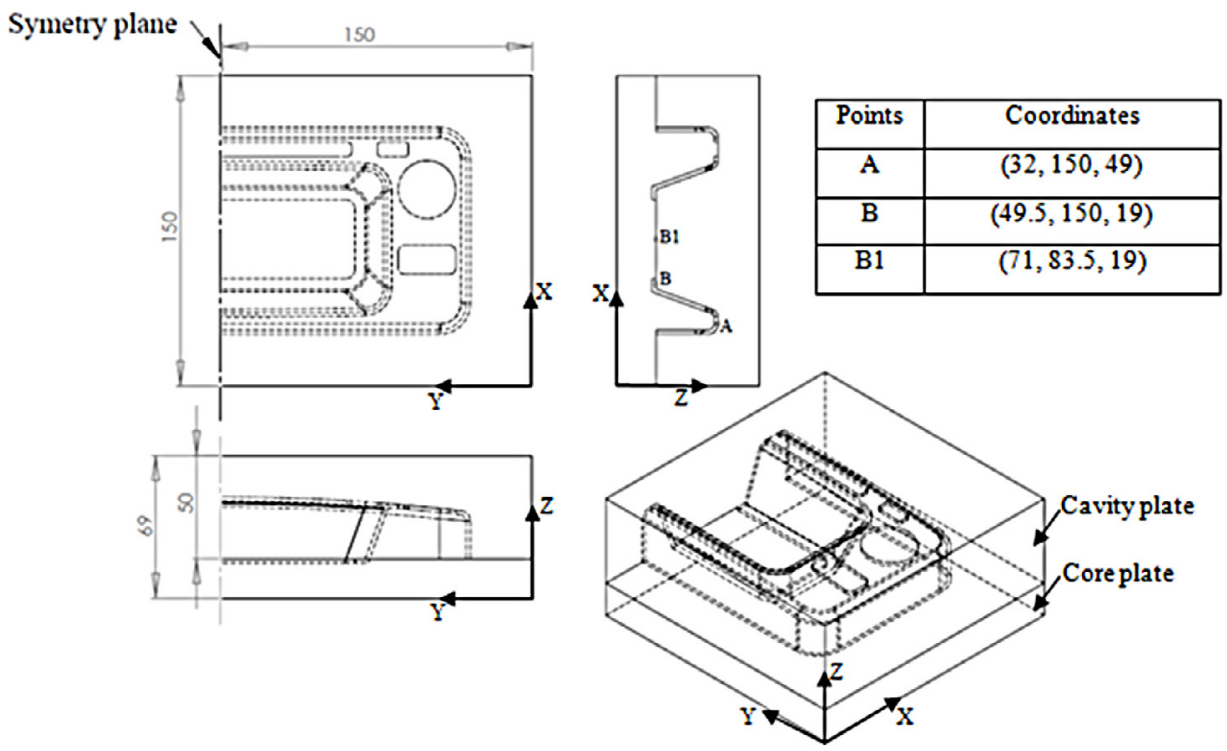

Fig. 2. Studied domain (half of the mold).

Table 1. Properties of mold material [26].

\begin{tabular}{cccc}
\hline & $\begin{array}{c}\text { Density } \\
\left(\mathrm{kg} \cdot \mathrm{m}^{-3}\right)\end{array}$ & $\begin{array}{c}\text { Calorific capacity } \\
\left(\mathrm{J} \cdot \mathrm{kg}^{-1} \cdot \mathrm{K}^{-1}\right)\end{array}$ & $\begin{array}{c}\text { Thermal conductivity } \\
\left(\mathrm{W} \cdot \mathrm{m}^{-1} \cdot \mathrm{K}^{-1}\right)\end{array}$ \\
\hline AMPCO 940 & 8710 & 380 & 208 \\
\hline
\end{tabular}

heating/cooling channels have been proposed. The importance of a new method, based on conventional manufacturing process which is able to produce complicated conformal heating/cooling channels with lower cost and easier fabrication, has been shown. Therefore, the numerical simulations of heat transfer in mold and cavity during a certain number of cycles are carried out.

\section{Mathematical formulation and numerical method}

This section presents the mathematical formulation and numerical method used to predict the thermal response in RHCM for the automotive part (Fig. 1a) production. Thermal responses, obtained with different channel layouts, are studied to minimize the RHCM cycle time and the temperature gap at the surfaces of the cavity plate that will generate outer functional surfaces of the product.

Initially, the mold (Fig. 2) made with AMPCO 940 [26] (Table 1) has an ambient temperature of $30^{\circ} \mathrm{C}$. The AMPCO copper-based alloys are characterized by its high thermal conductivity, and so, their uses increase significantly the production rates and the part quality. Before filling, the mold is heated. This stage finishes when the cavity functional surface exceeds the temperature of $140{ }^{\circ} \mathrm{C}$, which is assumed to be enough for the used polymer PC typed LEXAN Resin 915R [27] (Table 2). The cooling phase begins just after the filling stage and finishes when the polymer in the whole injected part is cooled until $120^{\circ} \mathrm{C}[27]$. At the end of the cooling stage, the ejection 
Table 2. Properties of the polymer [27].

\begin{tabular}{ccc}
\hline $\begin{array}{c}\text { Temperature } \\
\left({ }^{\circ} \mathrm{C}\right)\end{array}$ & $\begin{array}{c}\text { Calorific capacity } \\
\left(\mathrm{J}_{\mathrm{kg}}{ }^{-1} \cdot \mathrm{K}^{-1}\right)\end{array}$ & $\begin{array}{c}\text { Thermal conductivity } \\
\left(\mathrm{W} . \mathrm{m}^{-1} \cdot \mathrm{K}^{-1}\right)\end{array}$ \\
\hline 120 & 1612 & 0.275 \\
130 & 1693 & 0.278 \\
140 & 1823 & 0.282 \\
150 & 1885 & 0.286 \\
160 & 1909 & 0.298 \\
170 & 1929 & 0.31 \\
180 & 1947 & 0.322 \\
200 & 1976 & 0.227 \\
220 & 2003 & 0.235 \\
240 & 2020 & 0.242 \\
260 & 2030 & 0.248 \\
\hline
\end{tabular}

time is neglected and the obtained mold temperature distribution is used for the initial condition of the following molding cycle. So, a cyclic heating/cooling process was undertaken until attaining the steady cycle.

According to many authors $[15,20,21]$, the filling time of the mold cavity is usually short compared to the heating/cooling phase duration, and consequently, has little effect on the overall cycle. Based on this consideration, the filling time was neglected in this study. Consequently, at the end of the heating stage, the cavity is assumed to be filled with polymer at injection temperature and the cooling phase begins. Therefore, the heat transfer in the mold and polymer is purely conductive. The governing heat transfer Equation (1) related to the mold computational domain, shown in Figures $3 \mathrm{~b}, 9 \mathrm{~b}$ and $12 \mathrm{~b}$, is given by:

$$
\rho_{\mathrm{m}} C_{P_{\mathrm{m}}} \frac{\partial T_{\mathrm{m}}}{\partial t}=\nabla\left(\lambda_{\mathrm{m}} \nabla T_{\mathrm{m}}\right)
$$

For the computational polymer domain, represented in Figure 1b, heat transfer Equation (2) is given by:

$$
\rho_{\mathrm{p}} C_{P_{\mathrm{p}}}\left(T_{\mathrm{p}}\right) \frac{\partial T_{\mathrm{p}}}{\partial t}=\nabla\left(\lambda_{\mathrm{p}}\left(T_{\mathrm{p}}\right) \nabla T_{\mathrm{p}}\right)
$$

During the heating stage there is no polymer in the cavity, and the heat transfer is considered to occur only in the mold. During the cooling phase, the temperature effects on the thermal conductivity and specific heat of the polymer have been taken into account (Table 2). Furthermore, the used amorphous polymer Lexan PC 915R, has a low shrinkage (in the order of $0.5 \%$ to $0.8 \%$ ) [28] and then we have supposed a perfect contact at the interface moldpolymer and neglected the thermal contact resistance. So, the condition at the mold-polymer interface (Fig. 3c) is equality of temperature and continuity of heat flux:

$$
\left(\frac{\partial T_{\mathrm{m}}}{\partial n_{\mathrm{m}}}\right)_{\begin{array}{l}
\text { interfac } \\
\text { mold-polymer }
\end{array}}=-\left(\frac{\partial T_{\mathrm{p}}}{\partial n_{\mathrm{p}}}\right)_{\begin{array}{l}
\text { interface } \\
\text { mold-polymer }
\end{array}}
$$

For the RHCM process, due to the presence of insulating layers, heat transfer is only located at the cavity/core plates. Considering the symmetry of the studied part, it is sufficient to consider only half of the field (Fig. 2). For adiabatic surfaces (in contact with the insulation) and at the symmetry plane, we define a zero heat flux condition.

For the temperature control medium, the mold is equipped with channels in which the saturated steam circulates at $T_{\mathrm{s}}=180{ }^{\circ} \mathrm{C}$ during the heating phase, and the cooling water at $T_{\mathrm{w}}=30{ }^{\circ} \mathrm{C}$ throughout the cooling stage. The boundary conditions for the heat transfer interface mold-channels (Figs. 3d, 9c and 12c) introduce a heat transfer coefficients: $h_{\mathrm{h}}$ for the heating stage (4) and $h_{\mathrm{c}}$ for the cooling stage $(5)$.

$\lambda_{\mathrm{m}}\left(\frac{\partial T_{\mathrm{m}}}{\partial n_{\mathrm{m}}}\right)_{\begin{array}{l}\text { interface } \\ \text { mold-channels }\end{array}}=h_{\mathrm{h}}\left(T_{\mathrm{s}}-T_{\mathrm{m}}\right) \begin{aligned} & \text { interface } \\ & \text { mold-channels }\end{aligned}$

$$
\lambda_{\mathrm{m}}\left(\frac{\partial T_{\mathrm{m}}}{\partial n_{\mathrm{m}}}\right)_{\begin{array}{l}
\text { interface } \\
\text { mold-channels }
\end{array}}=h_{\mathrm{c}}\left(T_{\mathrm{w}}-T_{\mathrm{m}}\right)_{\begin{array}{l}
\text { interface } \\
\text { mold-channels }
\end{array}}
$$

For the heating stage, the coefficient of convective heat transfer for the vertical heating channels is obtained from the Nusselt condensation theory for the laminar flow of a falling condensate film in the inner tube of height $L$ [29]:

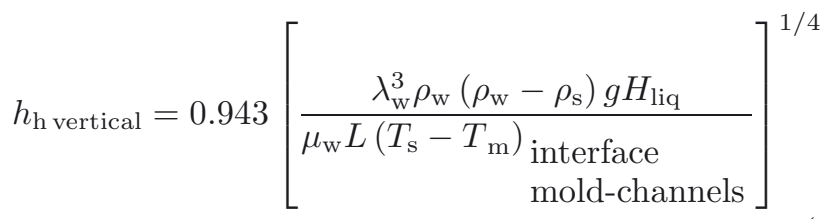

Rohsenow and Hartnett [29] derived a similar equation for film condensation on horizontal tubes giving a means heat transfers coefficient for a single horizontal tube of diameter $D$ :

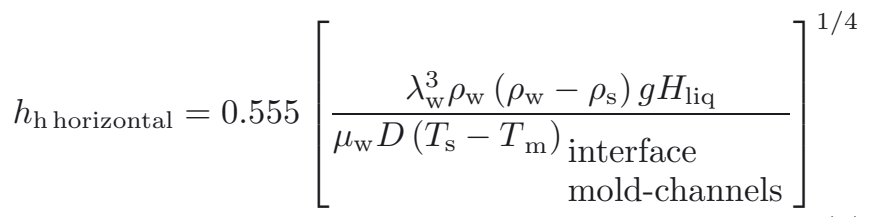

During the cooling phase, the heat transfer coefficient by convection is defined by [30]:

$$
h_{\mathrm{c}}=\left(5+0.015 \operatorname{Re}^{a} \operatorname{Pr}^{b}\right) \frac{\lambda_{\mathrm{w}}}{D}
$$

with: $a=0.88-\frac{0.24}{4+P r}$ and $b=0.333+0.5 \exp (-0.6 P r)$.

It is worthwhile to mention that for the studied configurations, the velocity of cold water is $0.6 \mathrm{~m} . \mathrm{s}^{-1}$.

Gambit 2.3.16 has been used to generate the grid for the CFD solver. Afterward, the previous governing differential equations have been solved numerically using the commercial software Fluent 6.3.26, which is based on a finite volume method. A pressure based implicit solver has been used to obtain thermal behaviors of mold and polymer through a certain number of cycles in order to reach the regular cyclic regime. The flowchart shown in 

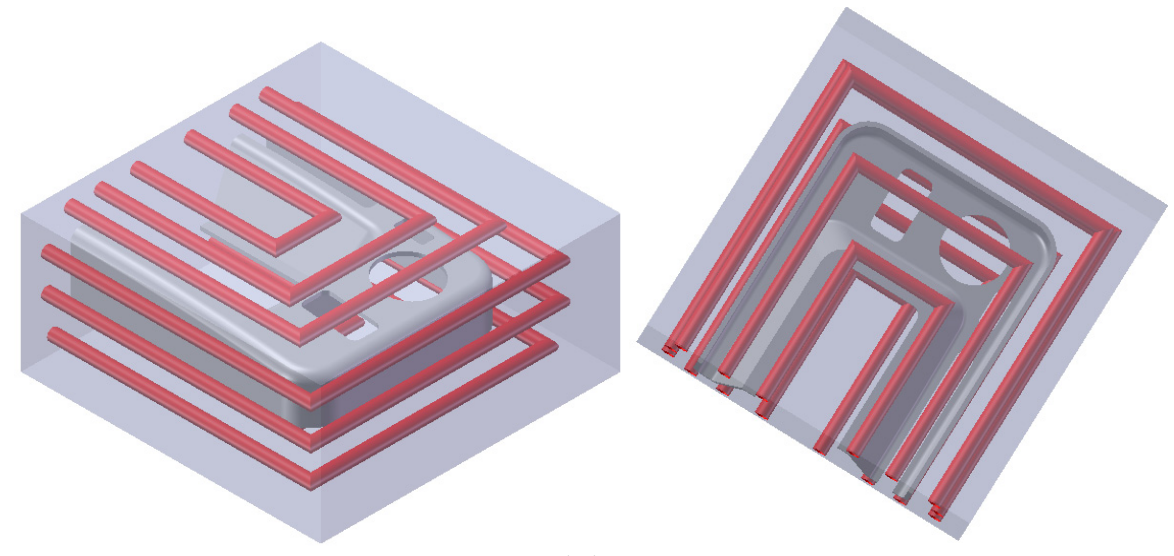

(a)

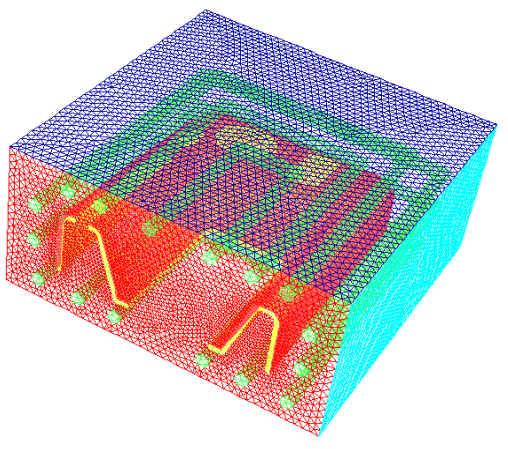

(b)

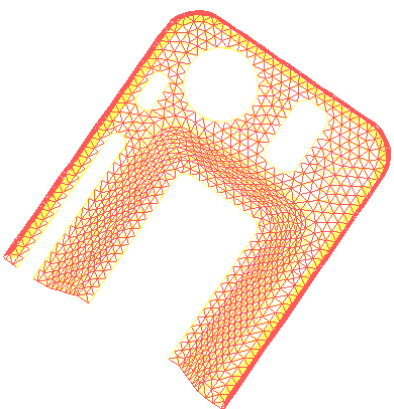

(c)

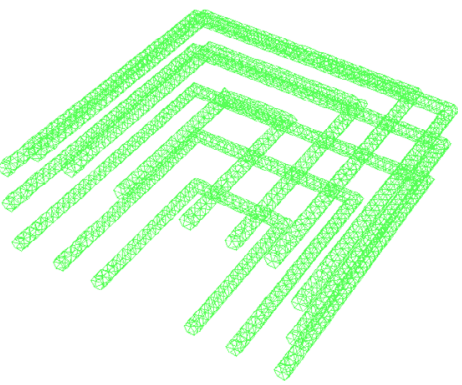

(d)

Fig. 3. Classic conformal design. (a) 3D model of $1 / 2$ RHCM mold, (b) grid of the mold domain, (c) Interface mold-polymer, (d) Interface mold- channels.

Figure 4 summarizes the numerical computation methodology used to simulate different cycles of the RHCM process.

A grid independence study is conducted employing fine, medium and coarse grids corresponding to the part and the mold domains. Temperature gap, heating and cooling times were used as key selection criteria in the grid independence study. The particulars of the grids and corresponding retained criteria are given in Table 3 . The best compromise between computational cost and result quality appears to be medium grid.

Furthermore, to prove the time step independence, three time steps are tested: $10^{-2} \mathrm{~s}, 10^{-3} \mathrm{~s}$ and $10^{-4} \mathrm{~s}$. Results shown in Table 4 prove that a time step of $10^{-3} \mathrm{~s}$ can reduce the computational time and it was sufficient to provide accurate results.

\section{Results and discussion}

\subsection{Classic conformal thermal control system}

Generally, the parallel straight heating/cooling channels cannot produce uniform cavity surface heating [22]. Then, for the first thermal control system, we propose a classic conformal design composed of horizontal channels following the general shape of the automotive part as shown in Figure 3a. The used unstructured grid into rectangular coordinates is composed by 2602007 tetrahedral elements: 1948032 elements for the mold and 653975 elements for the polymer.

Figure 5 shows the temperature distribution at the outer surfaces of the part at the end of the heating phase of the first, second and third molding cycle at $t=27.6 \mathrm{~s}$, $63.16 \mathrm{~s}$ and $95.4 \mathrm{~s}$, respectively. It should be noted that the end of the heating phases of each cycle is reached when a minimum temperature of $140{ }^{\circ} \mathrm{C}$ at the outer surfaces of the automotive part is attained. It can be seen from Figure 5 that we obtain the same temperature distribution at the end of the heating stage of the second and third molding cycle. Thus, a regular state is reached from the second molding cycle. Furthermore, it is demonstrated that for the first molding cycle, we obtain the most important cycle time of $48.25 \mathrm{~s}$ and temperature gap of $27.4^{\circ} \mathrm{C}$. Then, from the second molding cycle, constant values were reached (33 s for cycle time and $21^{\circ} \mathrm{C}$ for temperature gap).

To explain the origin of this difference between the first cycle and the regular one, the temperature maps given in Figure 6 show the temperature distribution at the symmetry plane at the end of the cooling phase for regular molding cycle. Since the ejection time is too shorten and can be neglected, the mold heating begins at the end of 
F. Kria et al.: Mechanics \& Industry 18, 109 (2017)

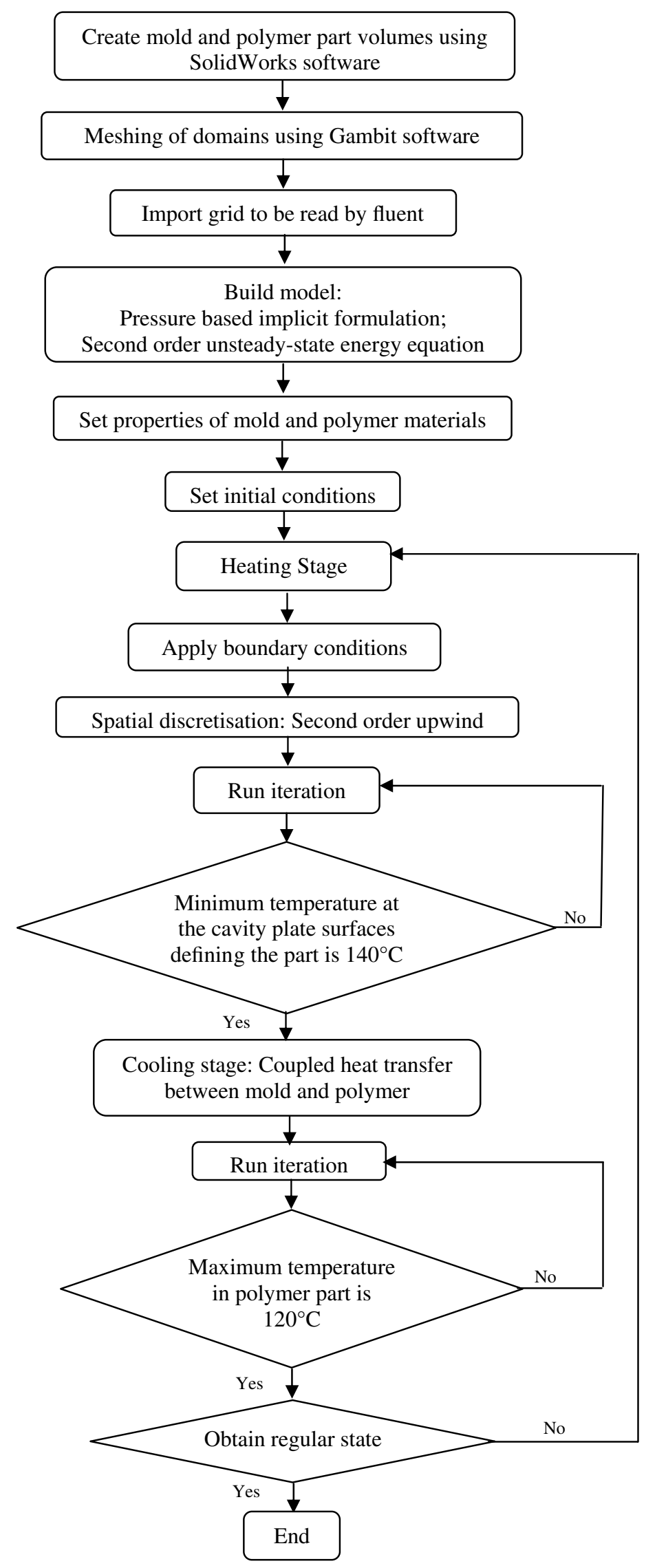

Fig. 4. Flowchart of computations. 
F. Kria et al.: Mechanics \& Industry 18, 109 (2017)

Table 3. Grid independence test.

\begin{tabular}{cccccc}
\hline & \multicolumn{2}{c}{ Grid elements } & \multirow{2}{*}{ Temperature gap $\left({ }^{\circ} \mathrm{C}\right)$} & \multirow{2}{*}{ Heating time $(\mathrm{s})$} & Cooling time $(\mathrm{s})$ \\
\hline Fine grid & Polymer domain & Mold domain & & 27.6 & 20.64 \\
Medium grid & 1033637 & 2721672 & 27.4 & 27.6 & 20.65 \\
Coarse grid & 703975 & 1948032 & 27.4 & 29.35 & 22.1 \\
\hline
\end{tabular}

Table 4. Time step independence test.

\begin{tabular}{lccc}
\hline & Temperature gap $\left({ }^{\circ} \mathrm{C}\right)$ & Heating time $(\mathrm{s})$ & Cooling time $(\mathrm{s})$ \\
\hline Time step 1: $10^{-4} \mathrm{~s}$ & 27.4 & 27.6 & 20.65 \\
Time step 2: $10^{-3} \mathrm{~s}$ & 27.4 & 27.6 & 20.65 \\
Time step 3: $10^{-2} \mathrm{~s}$ & 28 & 29.5 & 23.1 \\
\hline
\end{tabular}

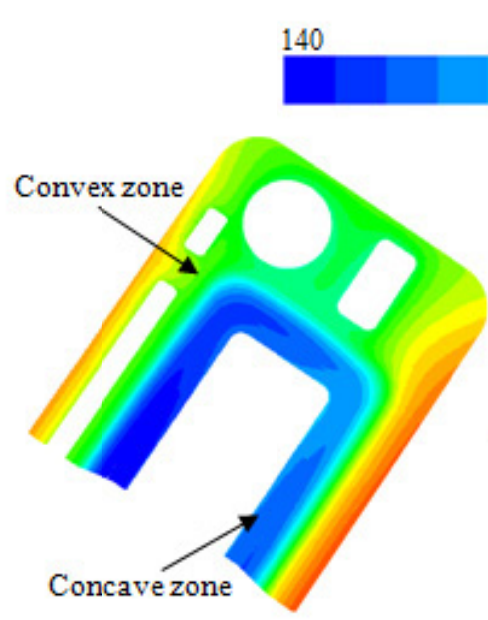

(a)
150

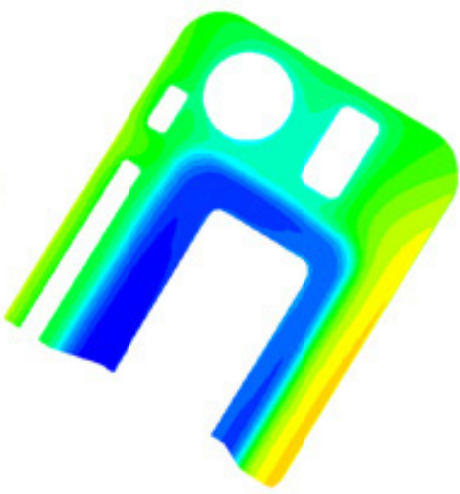

(b)
160

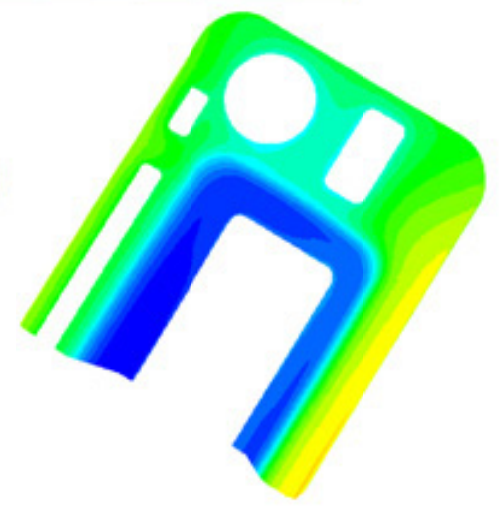

(c)

Fig. 5. Temperature distribution at the outer surfaces of the part with classic conformal design: (a) $t=27.6 \mathrm{~s}$ (end of the heating of the first cycle), (b) $t=63.16 \mathrm{~s}$ (end of the heating of the second cycle) and (c) $t=95.41 \mathrm{~s}$ (end of the heating of the third cycle).

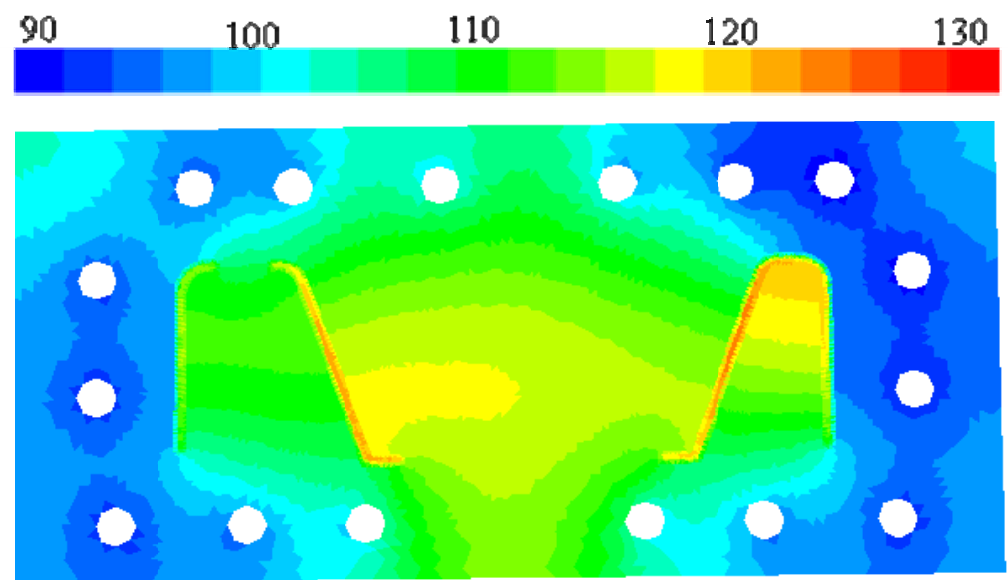

Fig. 6. Temperature distribution at the symmetry plane at $t=81.33 \mathrm{~s}$ (end of the cooling phase of the second cycle). 


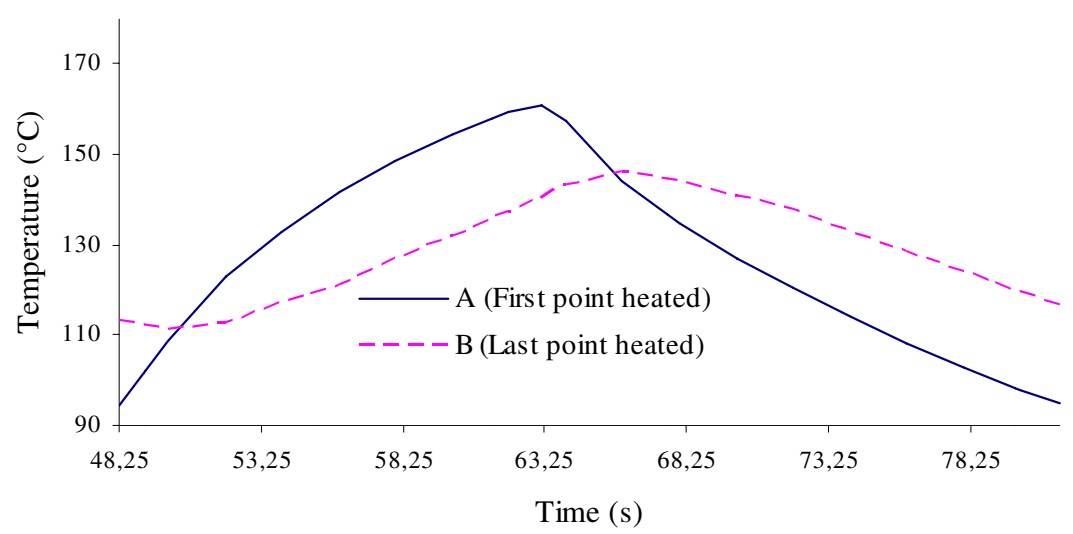

Fig. 7. Temperature evolution during the second molding cycle for classic conformal design.

the cooling stage. Figure 6 indiquates that the temperature at the central portion of the mold was relatively high $\left(115^{\circ} \mathrm{C}\right)$ and far from the considered initial temperature for the first cycle $\left(30^{\circ} \mathrm{C}\right)$. Therefore, this central zone allows the accumulation of thermal inertia for subsequent cycles, which can explain the reduction of the cycle time and the temperature gap.

Moreover, as shown in Figure 5, the cavity surface is divided into two regions during the heating process: concave areas with low heating rate and convex areas with high heating rate. These two areas lead to an unevenness temperature distribution generating several problems on the final product quality. Figure 7 further clarifies the situation and reveals the temperature temporal evolution during the second molding cycle for the first (A) and last (B) heated points (Figs. 1 and 2) which determine the maximal temperature gap.

It can be seen that the temperature variation is more significant at the first heated point located at the convex areas of the part, than that at the last point heated situated at the concave areas. The low heating/cooling rate at these areas is caused by the large distance between the heating/cooling channels and the concave portions of the part. Therefore, it is impossible to achieve a rapid and uniform temperature distribution. For this reason, improving the design of the heating/cooling channels is necessary to ensure the efficiency of the RHCM process.

\subsection{New conformal thermal control system}

To improve heat transfer at the convex areas of cavity/core plates, a new conformal design of heating/cooling channels has been proposed. In the present studied design, besides the horizontal channels, vertical independent channels are added to reach the concave regions of the automotive part and ensure uniform temperature along the mold surface. With this proposed strategy, heat transfer at concave zones can be improved and consequently, the heating/cooling rate can be accelerated and the temperature gap between concave and convex areas can be attenuated. With this new method, the mold has the advantages of being easy to manufacture and assemble, at the same time; it is cheaper than other methods producing conformal systems like $3 \mathrm{D}$ printing $[4,6,7]$. In fact, simple drillings associated with concentric tubes are able to generate conformal heating/cooling channels for RHCM process producing complex shaped automotive part.

The principle of this proposed new design is shown in Figure 8. In fact, the heating/cooling fluid enters through a main channel and passes through a vertical tube, in which there will be a distribution of fluid to the principal drilled hole which is concentric with the vertical tube.

\subsubsection{First new conformal design}

In order to remedy to the temperature significant gap obtained with the classic design formed only with horizontal channels, a new conformal design is proposed. With this proposed design, number of heating/cooling channels should be vertically positioned to reach concave regions of the part and then to enhance the heat exchange.

As a first new conformal heating/cooling channels design, we proposed the system shown in Figure 9a. At the cavity plate, this system includes $(6 \times 2)$ concentric vertical channels at the central convex zone. The main drilling is $8 \mathrm{~mm}$ in diameter and $43 \mathrm{~mm}$ in length. Also, it contains 4 parallel straight horizontal channels and 2 conformal horizontal channels of $6 \mathrm{~mm}$ in diameter. For the core plate, the heating/cooling system encloses $(3 \times 2)$ concentric vertical channels distributed as follows: $(3 \times 2)$ vertical drillings of $14 \mathrm{~mm}$ in diameter and $28 \mathrm{~mm}$ in length, $(3 \times 2)$ vertical drillings of $8 \mathrm{~mm}$ in diameter and $30 \mathrm{~mm}$ in length and $(3 \times 2)$ vertical drillings of $6 \mathrm{~mm}$ in diameter and $28 \mathrm{~mm}$ in length. The mesh adopted for the computational domain contains 2346700 tetrahedral elements divided as follows (653975 elements in polymer field and 1692725 elements in mold domain).

The numerical simulations of the three first cycles show that in a regular state, the cycle time is $28.5 \mathrm{~s}$. A reduction of $13.6 \%$ is then attained with this new design. Concerning the temperature distribution, Figure 10 illustrates the temperature maps at the end of the heating phase of the first, second and third cycle, reproduced 


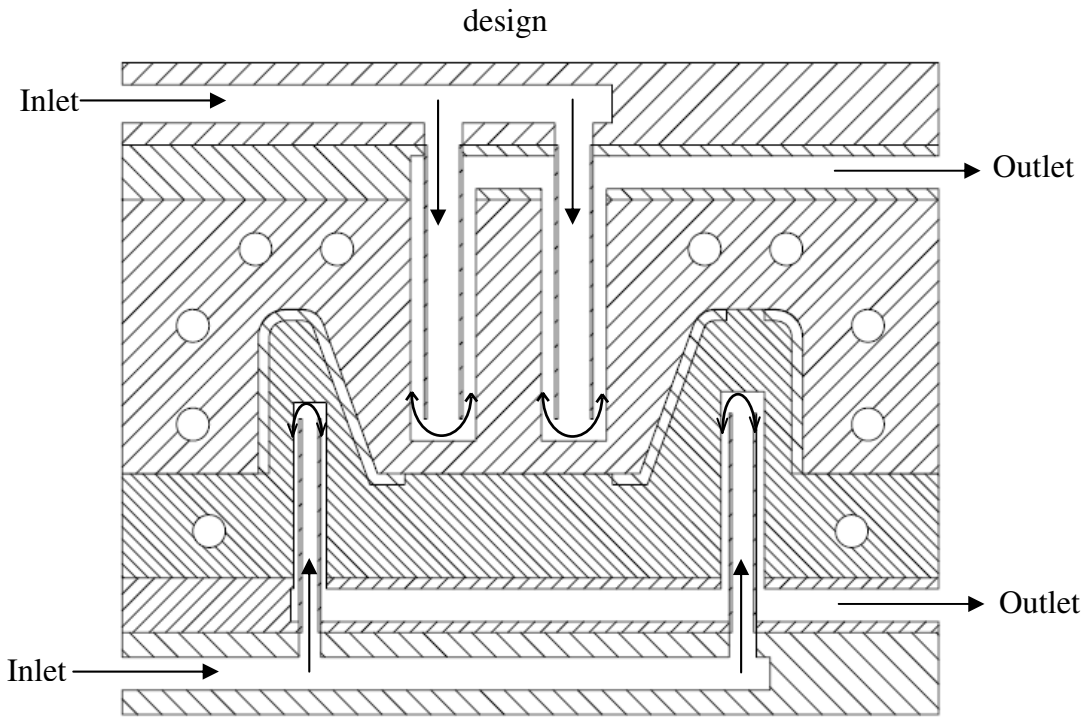

Fig. 8. Principle of heating/cooling channels design.

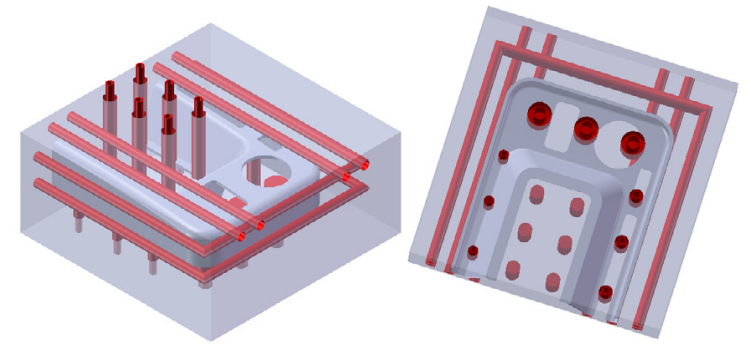

(a)

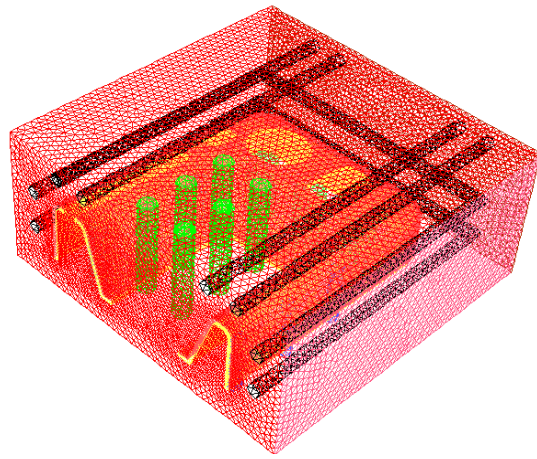

(b)

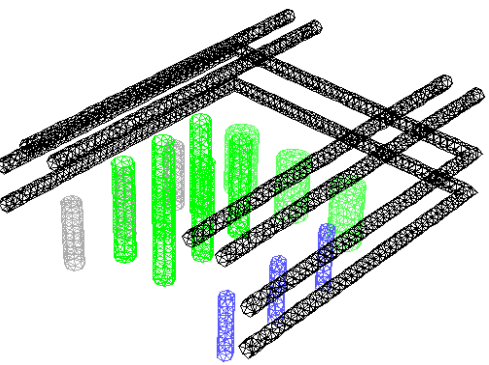

(c)

Fig. 9. First new conformal design. (a) 3D model of $1 / 2 \mathrm{RHCM}$ mold, (b) the grid of mold computational domain, (c) interface mold-channels.

respectively at $t=24.52 \mathrm{~s}, 54.63 \mathrm{~s}$ and $83.2 \mathrm{~s}$. Comparing the thermal responses of the classic conformal design (Fig. 5) to those obtained with the first new design (Fig. 10), we can note a significant reduction of the cold area at the cavity surface. However, the temperature maps of Figure 10 prove again an uneven temperature distribution at the end of heating phase, which can be verified by a temperature gap of $19.7^{\circ} \mathrm{C}$.

Figure 11 shows the time evolution of the temperature during the second heating/cooling cycle for the first (A) and the last (B) heated points (Figs. 1 and 2).
Even with this new design, we can note a significant difference between temporal profiles reproducing temperature evolutions for the two chosen points. Compared to the first classic conformal system, there is only slightly attenuation of temperature gap, which can be explained by an insufficient heat surface exchange offered by vertical channels. Thus, the temperature disparity between the concave and convex areas of the automotive part has not yet been resolved.

This new conformal design represents an improvement especially at the cycle time. However, as it was not 


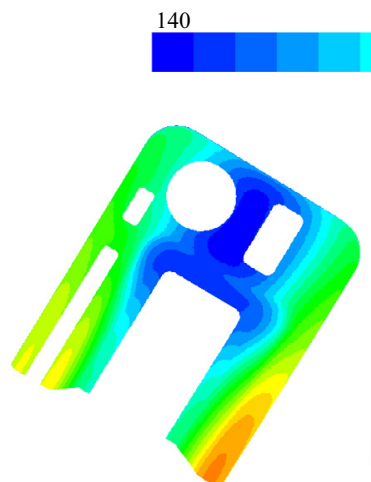

(a)
150

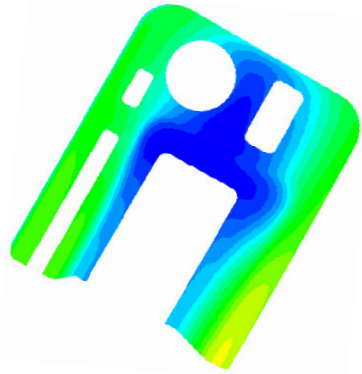

(b)
160

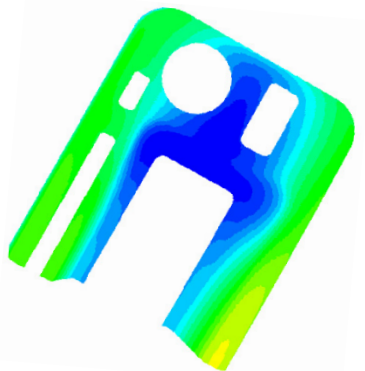

(c)

Fig. 10. Temperature distribution at the outer surfaces of the part with first new design: (a) $t=24.52 \mathrm{~s}$ (end of the heating stage of the first cycle), (b) $t=54.63 \mathrm{~s}$ (end of the heating stage of the second cycle) and (c) $t=83.2 \mathrm{~s}$ (end of the heating stage of the third cycle).

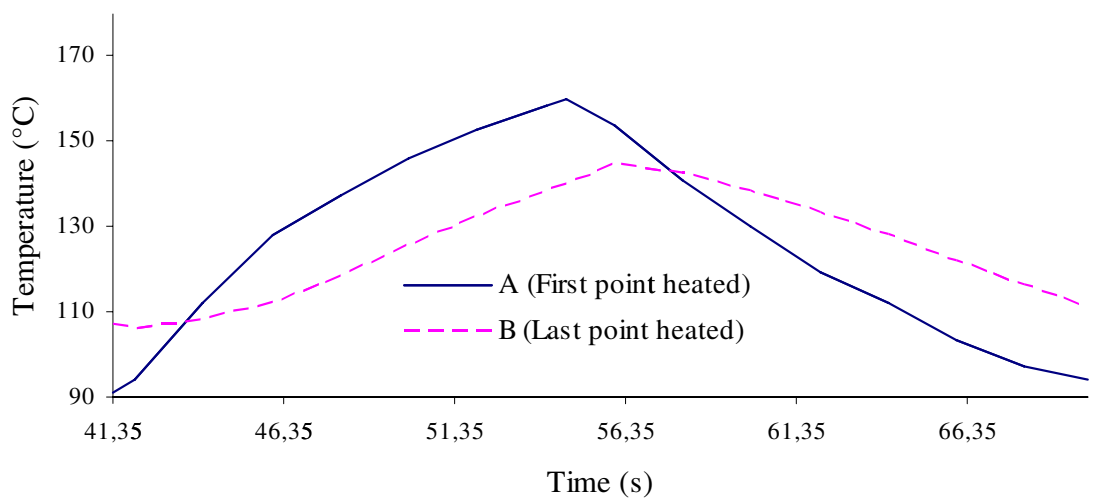

Fig. 11. Temperature evolution during the second molding cycle for first new conformal design.

sufficient to ensure product quality and process productivity, a second new conformal design has been proposed.

\subsubsection{Second new conformal design}

After several attempts, a second design described in Figure 12a was proposed. In order to improve heat transfer at convex surface of the cavity plate, an increase of the external diameter of vertical channels has been adopted.

For the cavity plate, the system has $(6 \times 2)$ vertical channels of $12 \mathrm{~mm}$ in diameter and $44 \mathrm{~mm}$ in length. For the core plate, the position and number of vertical heating/cooling channels were kept the same. The adopted mesh of the computational domain contains 2447300 tetrahedral elements distributed as follows (653975 elements in polymer field and 1793325 elements in mold domain).

The temperature maps of Figure 13 give the overall appearance of the temperature distribution at the end of the heating phases of the studied cycles at $t=18.62 \mathrm{~s}$, $38.85 \mathrm{~s}$ and $59.68 \mathrm{~s}$. Compared to thermal responses acquired with the first new design (Fig. 10), the second new design (Fig. 13) shows improvement of the heating rate in both concave and convex zones. We also notice that these zones reach the necessary temperature $\left(140^{\circ} \mathrm{C}\right)$ nearly at the same time giving uniform temperature distribution. Consequently, the second design leads to regulation of the temperature of the RHCM mold in a reduced cycle time $(20.8 \mathrm{~s})$ with a lower temperature gap $\left(13^{\circ} \mathrm{C}\right)$.

Figure 14 presents a comparison of the temporal evolution of temperature for the first (A) and the last (B1) heated point (Figs. 1 and 2). It can be seen that the temperature variation for these points are almost the same for both heating and cooling phases. Therefore, the second new design allows a significant improvement in the temperature distribution between the concave and convex surfaces of the studied part.

\subsection{Comparison of the three systems}

Figure 15 represents the temperature cyclic evolution of point A (First heated point) (Figs. 1 and 2) during the three first molding cycles for the three proposed systems. It has been found that the regular state is reached rapidly for the RHCM process compared with CIM. The addition of concentric vertical channels at the convex areas of cavity/core plates promotes heat transfer to the convex zones 
F. Kria et al.: Mechanics \& Industry 18, 109 (2017)
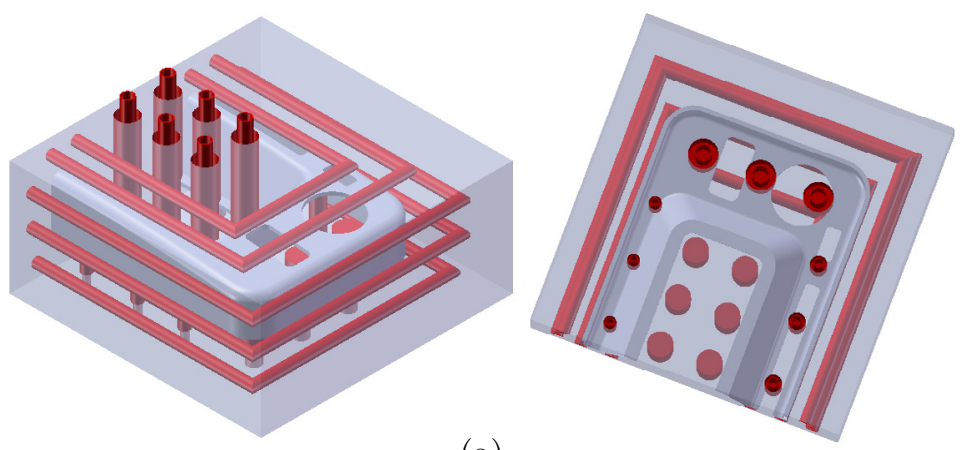

(a)

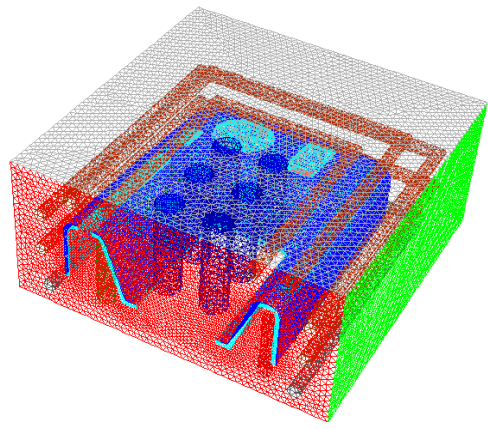

(b)

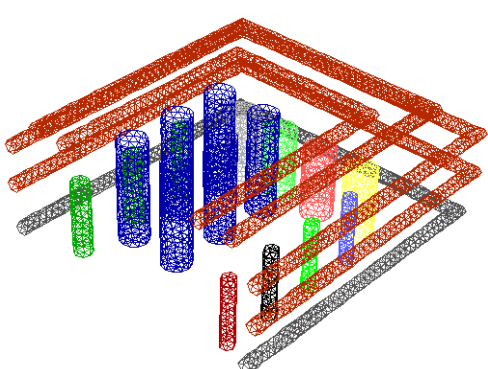

(c)

Fig. 12. Second new conformal design. (a) 3D model of $1 / 2$ RHCM mold, (b) the grid of mold computational domain, (c) interface mold-polymer.

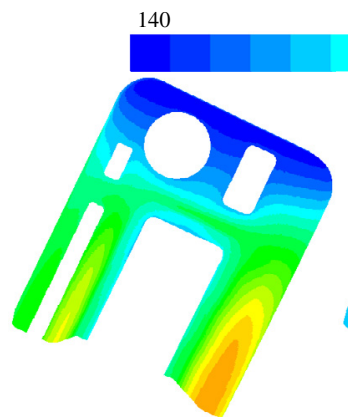

(a)

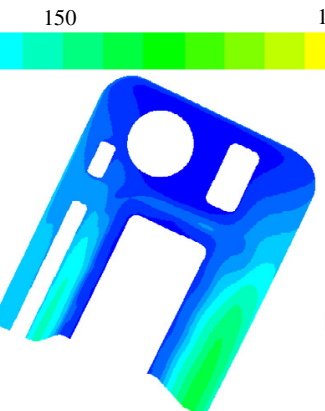

(b)
160

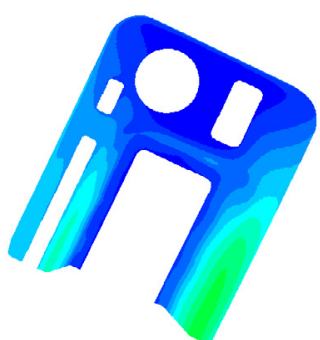

(c)

Fig. 13. Temperature distribution at the outer surfaces of the part with second new design: (a) $t=18.62 \mathrm{~s}$ (end of the heating of the first cycle), (b) $t=38.85 \mathrm{~s}$ (end of the heating of the second cycle) and (c) $t=59.68 \mathrm{~s}$ (end of the heating of the third cycle).

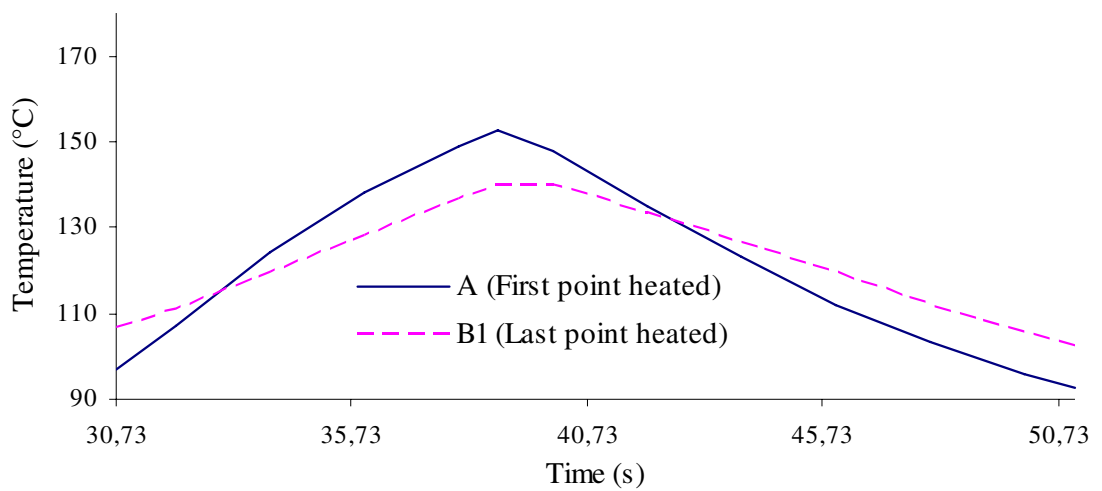

Fig. 14. Temperature evolution during the second molding cycle for second new conformal design. 


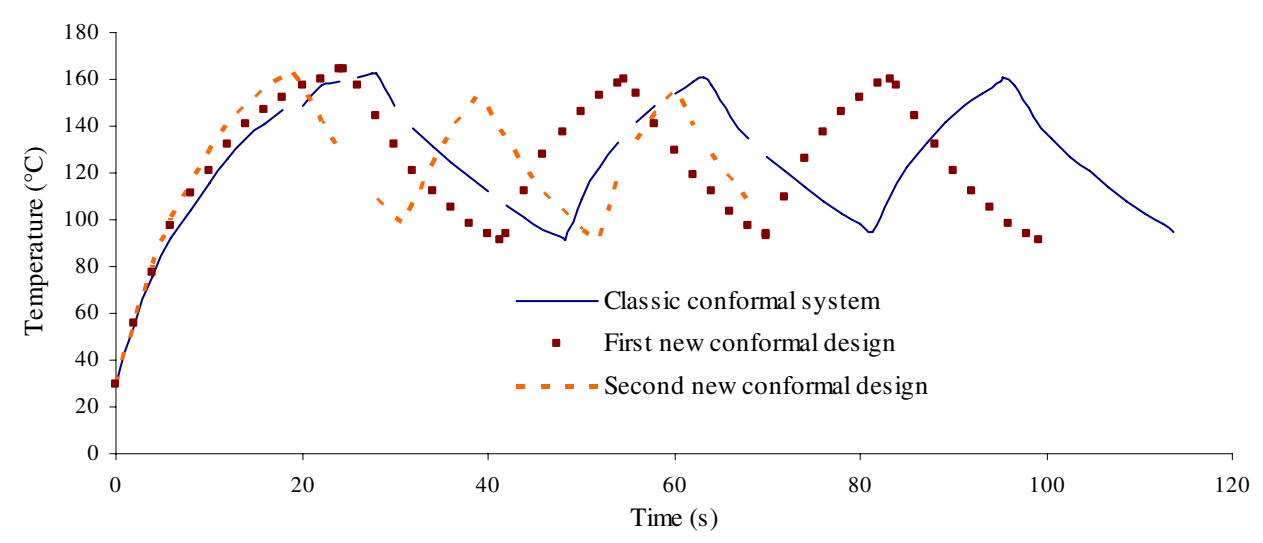

Fig. 15. Temperature evolution of point A (first point heated).

of the cavity/core plates. Furthermore, the temperature of point (A) for the regular state is greater than the ambient temperature. This may explain the reduction of the cycle time for the following cycles.

To further inquire the differences between the suggested thermal control designs, the histograms presented in Figure 16 compare the heating/cooling time, temperature gap and consumed energy for the three first molding cycles.

Comparing the classic conformal system with the first new design, an acceptable reduction can be seen in the cycle time. However, the improvements in the temperature distribution still remain very low and insufficient to ensure a good surface state. For the second new design, a significant reduction in the cycle time and temperature gap can be observed. Similarly, a reduction in the quantity of consumed energy is noted to this configuration. This indicates the effectiveness of the second new design of RHCM process producing automotive part.

\section{Conclusion}

A 3D numerical study was performed to investigate the heat transfer during the heating and cooling phases of the RHCM process for complex shaped automotive part production. Two types of conformal thermal control systems were studied. The classic conformal system composed only of horizontal channels following the general shape of the part, and a new conformal design, incorporating vertical channels fitting the concave areas of the complex shaped automotive part.

In order to reach the regular cyclic regime, multiple molding cycles were simulated. We have registered an important difference in terms of cycle time and consumed energy, between the first molding cycle and the following ones. Moreover, it has been demonstrated that:

- The stable cyclic regime is established from the second molding cycle.

- The classic conformal system has many limitations. Actually, it has been proven that the heating/cooling rate at the concave areas of the part is very low com-

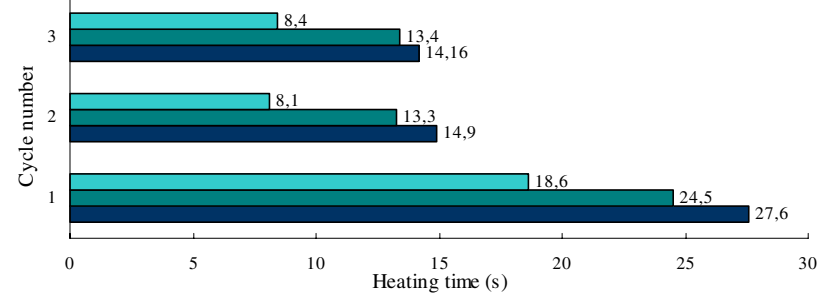

(a) Heating stage

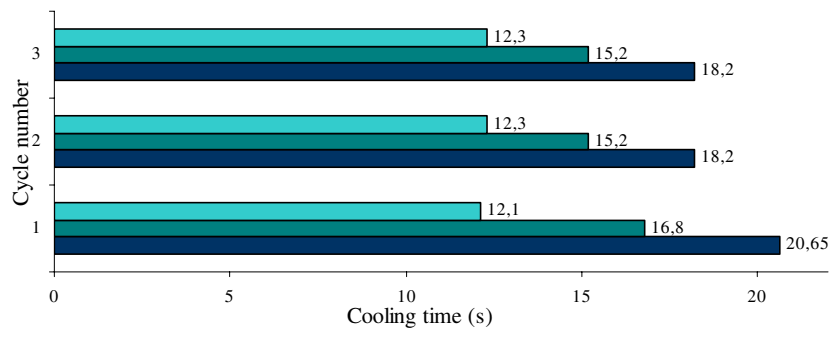

(b) Cooling stage

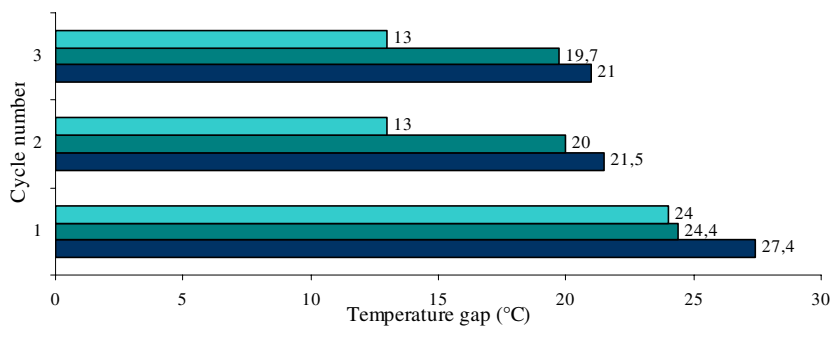

(c) Temperature gap

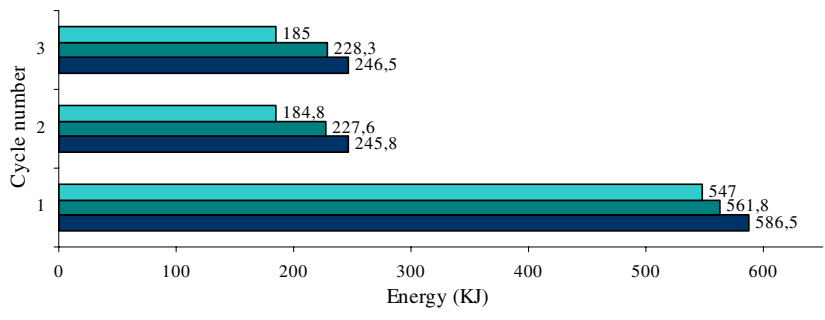

(d) Consumed energie

$\square$ Second new desig

$\square$ First new design

Classic conformal system

Fig. 16. Comparison of the studied systems. (a) Heating stage, (b) Cooling stage, (c) Temperature gap, (d) Consumed energie. 
pared with the rate at other part surfaces, which generates an uneven temperature distribution.

- Based on a conventional machining process, the production of conformal heating/cooling channels for the complex shaped part is possible. Indeed, by combining simple drillings with concentric tubes, the expensive methods producing complicated conformal channels can be overcome.

- The second new design decreases the cycle time by $37 \%$, temperature gap by $38.1 \%$ and consumed energy by $25 \%$.

\section{References}

[1] H. Hassan, N. Regnier, C. Lebot, C. Pujos, G. Defaye, Effect of cooling system on the polymer temperature and solidification during injection molding, Appl. Thermal Eng. 29 (2009) 1786-1791

[2] A. Agazzi, V. Sobotka, R. LeGoff, Y. Jarny, Optimal cooling design in injection moulding process - A new approach based on morphological surfaces, Appl. Thermal Eng. 52 (2013) 170-178

[3] Z. Shayfull, S. Sharif, A. Mohd Zain, R. Mohd Saad, M.A. Fairuz, Milled groove square shape conformal cooling channels in injection molding process, Mater. Manuf. Process. 28 (2013) 884-891

[4] Y. Wang, Y. Kai-Min, C.L. Wang, Y. Zhang, Automatic design of conformal cooling circuits for rapid tooling, Comput. Aided Design 43 (2011) 1001-1010

[5] X.P Dang, H.S. Park, Design of u-shape milled groove conformal cooling channels for plastic injection mold, Int. J. Precis. Eng. Manuf. 12 (2011) 73-84

[6] J. Liu, Z. Lu, Y. Shi, W. Xu, J. Zhang, Investigation into manufacturing injection mold via indirect selective laser sintering, Int. J. Adv. Manuf. Technol. 48 (2010) 155-163

[7] D.-G. Ahn, Applications of laser assisted metal rapid tooling process to manufacture of molding and forming tools - state of the art, Int. J. Precis. Eng. Manuf. 12 (2011) 925-938

[8] R.Y. Chang, B.D. Tsaur, Experimental and theoretical studies of shrinkage, warpage and sink marks of crystalline polymer injection molded parts, Polymer Eng. Sci. 35 (1995) 1222-1230

[9] A. Daniele, R.-A. Jeffrey, Review of Factors that Affect Shrinkage of Molded Part in Injection Molding, Mater. Manuf. Process. 29 (2014) 662-682

[10] H. Hassan, N. Regnier, C. Pujos, E. Arquis, G. Defaye, Modeling the effect of cooling system on the shrinkage and temperature of the polymer by injection molding, Appl. Thermal Eng. 30 (2010) 1547-1557

[11] G. Wang, G. Zhao, Y. Guan, Thermal response of an electric heating rapid cycle molding mold and its effect on surface appearance and tensile strength of the molded part, J. Appl. Polymer Sci. 128 (2013) 1339-1352

[12] G. Wang, G. Zhao, X. Wang, Experimental research on the effects of cavity surface temperature on surface appearance properties of the molded part in rapid heat cycle molding process, Int. J. Adv. Manuf. Technol. 68 (2013) $1293-1310$
[13] G. Lucchetta, M. Fiorotto, P.F. Bariani, Influence of rapid mold temperature variation on surface topography replication and appearance of injection-molded parts, CIRP Annals - Manuf. Technol. 61 (2012) 539-542

[14] G. Lucchetta, M. Fiorotto, Influence of Rapid Mould Temperature Variation on the Appearance of InjectionMoulded Parts, J. Mech. Eng. 59 (2013) 683-68

[15] X.-P. Li, G.-Q. Zhao, Y.-J. Guan, M.-X. Ma, Optimal design of heating channels for rapid heating cycle injection mold based on response surface and genetic algorithm, Mater. Design 30 (2009) 4317-4323

[16] G. Zhao, G.Wang, Y.Guan, H. Li, Research and application of a new rapid heat cycle molding with electric heating and coolant cooling to improve the surface quality of large LCD TV panels, Polymer Adv. Technol. 22 (2011) 476-487

[17] X.-P. Li, N.-N. Gong, Y.-J. Guan, G.-M. Cheng, Thermal and stress analysis of rapid electric heating injection mold for a large LCD TV panel, Appl. Thermal Eng. 31 (2011) 3989-3995

[18] P.-C. Chang, S.-J. Hwang, Experimental investigation of infrared rapid surface heating for injection molding, J. Appl. Polymer Sci. 102 (2006) 3704-3713

[19] M.-C. Jeng, S.-C. Chen, P. S. Minh, J.-A.Chang, C.S. Chung, Rapid mold temperature control in injection molding by using steam heating, Int. Commun. Heat Mass Transfer 37 (2010) 1295-1304

[20] G. Wang, G. Zhao, H. Li, Y. Guan, Research on optimization design of the heating/cooling channels for rapid heat cycle molding based on response surface methodology and constrained particle swarm optimization, Expert Syst. Appli. 38 (2011) 6705-6719

[21] M. Wang, J. Dong, W. Wang, J. Zhou, Z. Dai, X. Zhuang, X. Yao, Optimal design of medium channels for water-assisted rapid thermal cycle mold using multiobjective evolutionary algorithm and multi-attribute decision-making method, Int. J. Adv. Manuf. Technol. 68 (2013) 2407-2417

[22] M. Hammami, F. Kria , M. Baccar, Numerical study of thermal control system for rapid heat cycle injection molding process, J. Process Mech. Eng. Part E. 229 (2015) 315-326

[23] G. Wang, G. Zhao, X. Wang, Effects of cavity surface temperature on mechanical properties of specimens with and without a weld line in rapid heat cycle molding, Mater. Design 46 (2013) 457-47

[24] C.-L. Xiao, H.-X. Huang, Development of a rapid thermal cycling molding with electric heating and water impingement cooling for injection molding applications, Appl. Thermal Eng. 73 (2014) 712-722

[25] G. Wang, G. Zhao, X. Wang, Heating/cooling channels design for an automotive interior part and its evaluation in rapid heat cycle molding, Mater. Design 9 (2014) 310322

[26] Moldflow plastic insight release 5.0, 2002

[27] Autodesk simulation Moldflow adviser, 2014

[28] Technical engineering A $3381-8$

[29] M.W. Rohsenow, J.P. Hartnett, Handbook of heat transfer, McGraw-Hill, New York, 1973

[30] C.-A. Sleicher, M.-W. Rousse, A convenient correlation for heat transfer to constant and variable property fluids in turbulent pipe flow, Int. J. Heat Mass Transfer 18 (1975) 677-683 